\section{Ewing's sarcoma with distant metastasis: A brief note on management and emerging therapies}

Girish Gulab Meshram, ${ }^{1}$ Neeraj Kaur, ${ }^{2}$ Kanwaljeet Singh Hura ${ }^{3}$

${ }^{1}$ Department of Pharmacology, Postgraduate Institute of Medical Education and Research and Dr. Ram Manohar Lohia Hospital, New Delhi, India; ${ }^{2}$ Department of Radiology, University of Texas Health Science Centre, San Antonio, Texas, USA; ${ }^{3}$ Department of Pediatrics, Richmond University Medical Centre, Staten Island, New York, USA

\begin{abstract}
Ewing's sarcoma is an aggressive fatal malignancy of bones and soft-tissue. It predominantly affects the young population, with a worldwide incidence of three cases per million. The pelvis, extremities, and ribs are the most common sites. We present a case of massive Ewing's sarcoma of the right femur with metastasis to bones and lungs. The patient was treated with chemotherapy. However, he succumbed to his illness before completion of therapy. In conclusion, Ewing's sarcoma with distant metastasis is a high risk case with poor prognosis. Integrating novel molecular targets with conventional chemotherapeutic agents holds a promise for high-risk Ewing's sarcoma patients.
\end{abstract}

\section{Introduction}

Ewing's sarcoma is an aggressive bone or soft-tissue sarcoma that primarily afflicts children and adolescents, with peak occurrence between ages 10 and $20 .{ }^{1}$ Fusion between the 5' segment of the Ewing sarcoma breakpoint region 1 (EWSR1) gene and the 3' portion of the Friend leukemia virus integration site 1 (FLI1) gene is detected in around $85 \%$ cases of Ewing's sarcoma. ${ }^{2}$ Other rarer translocation abnormalities have also been implicated in the pathogenesis of Ewing's sarcoma. Translocations give rise to chimeric genes, which act as aberrant transcription factors and promote tumorigenesis. ${ }^{3}$ Common sites of primary disease include pelvis, ribs, and long-bone extremities. ${ }^{4}$ Approximately $20-25 \%$ of patients present with metastasis at diagnosis. ${ }^{5} \mathrm{We}$ present a case of massive Ewing's sarcoma of the right femur in a 5-year-old child with metastasis to the lungs and bones. We have also briefly discussed the novel therapeutic agents in the pipeline.

\section{Case Report}

A 5-year-old child presented to the department of pediatrics with a swelling at his right lower limb. The swelling was first noticed around 10 months back and had been gradually increasing since then. Pain at the site of the swelling was initially intermittent but had become persistent since the past 5 months. In addition, the patient also complained of fever, weight loss, and nonproductive cough. There was no history of trauma, fever, and night sweats. General examination was unremarkable except for the presence of mild pallor. On local examination, the size of the swelling was around $25 \mathrm{~cm}$ in height and had a circumference of around $40 \mathrm{~cm}$ (Figures 1 and 2). Blood investigations revealed leukocytosis and raised erythrocyte sedimentation rate. Tests for tuberculosis were negative. X-ray of the femur showed an ill-defined lytic lesion at the lower one-third of the femur with areas of bone destruction in center and periosteal reaction on the outer side. Magnetic resonance imaging on both $\mathrm{T} 1$ and $\mathrm{T} 2$ weighted signals showed an ill-defined mass of increased heterogeneity infiltrating into the adjacent tissue with surrounding edema. Fine-needle biopsy of the lesion revealed nests of small round hyperchromatic cells with increased nuclear-cytoplasmic ratio. Periodic acid-Schiff staining demonstrated the presence of intracytoplasmic glycogen. Immunohistochemical staining was positive for CD99 and was negative for antileukocyte common antigen, neuron specific enolase, and desmin. Bone scan showed secondary lesions at the upper one-third of the right femur, and $\mathrm{L}_{3}, \mathrm{~L}_{4}$ bodies. $\mathrm{X}$-ray of the chest appeared normal. High resolution computed tomography scan of the chest showed presence of two nodules at the left lower lobe, one at the left hilum, and two at the right lower lobe. Due to the limited affordability of the patient, we could not conduct molecular studies for identifying the underlying genetic abnormalities. Based on history, examination and investigations, a diagnosis of Ewing's sarcoma with pulmonary/bone metastasis was reached. Neoadjuvant chemotherapy consisting of 6 cycles of vincristine (V), adriamycin (D), actinomycin-D (A), cyclophosphamide (C) alternating with ifosfamide (I) and etoposide (E) was planned for the patient, followed by local wide resection and radio-
Correspondence: Girish Gulab Meshram, Department of Pharmacology, Postgraduate Institute of Medical Education, Research and Dr. Ram Manohar Lohia Hospital, New Delhi 110001, India.

Tel.: +91.8376010560

E-mail: drgirish23@yahoo.co.in

Key words: Ewing's sarcoma; Distant metastasis; Chemotherapy.

Contributions: the authors contributed equally.

Conflict of interest: the authors declare no potential conflict of interest.

Funding: none.

Received for publication: 29 October 2018

Revision received: 28 December 2018.

Accepted for publication: 17 January 2019.

This work is licensed under a Creative Commons Attribution NonCommercial 4.0 License (CC BY-NC 4.0).

(C) Copyright: the Author(s), 2019

Licensee PAGEPress, Italy

Clinics and Practice 2019; 9:1111

doi:10.4081/cp.2019.1111

therapy. The response to neoadjuvant chemotherapy was to be assessed histopathologically preoperatively, according to the Salzer-Kuntschik grading scale. However, at the end of the $2^{\text {nd }}$ cycle of chemotherapy the patient developed acute respiratory distress and succumbed to his illness.

\section{Discussion}

Ewing's sarcoma was first described by James Ewing in the year 1921. It is the second most common bone tumor in childhood and adolescence, after osteosarcoma. ${ }^{6}$ Although debatable, several reports indicate that the mesenchymal stem cells are the original progenitors of Ewing's sarcoma.? The molecular event which triggers Ewing's sarcoma is a chromosomal translocation, which fuses the EWSR1 gene to one of the five genes of the E twenty-six (ETS) family which includes FLI1, v-ets avian erythroblastosis virus E26 oncogene homolog (ERG), ETS variant 1 (ETV1), E1A enhancer binding protein (ETV4), and fifth Ewing sarcoma variant (FEV). ${ }^{3,8}$ These translocations are $\mathrm{t}(11 ; 22)(\mathrm{q} 24 ; \mathrm{q} 12)$, $\mathrm{t}(21 ; 22)(\mathrm{q} 22 ; \mathrm{q} 12), \quad \mathrm{t}(7 ; 22)(\mathrm{q} 22 ; \mathrm{q} 12)$, $\mathrm{t}(17 ; 22)(\mathrm{q} 21 ; \mathrm{q} 12)$, and $\mathrm{t}(2,22)(\mathrm{q} 35 ; \mathrm{q} 12)$ which give rise to the fusion genes EWSR1FLI1 (85\% of cases), EWSR1-ERG (10\% 
of cases), EWSR1-ETV1 ( $<1 \%$ of cases), EWSR1-ETV4 $(<1 \%$ of case), and EWS1FEV ( $<1 \%$ of cases), respectively. ${ }^{4}$ The resultant fusion gene functions as a constitutively active transcription factor, encodes a chimeric protein and regulates numerous other genes, responsible for the oncogenic behavior of Ewing's sarcoma. ${ }^{1-3}$

Patients usually present with a tumor mass or localized pain. Other systemic symptoms may include fever, malaise, and weight loss, as found in our patient. In western countries, the duration between the onset of the initial symptom and diagnosis is around 5 months. ${ }^{4}$ However, in our case it was around 10 months as the patient sought medical care from folkloric practitioners in his village.

Blood investigations may reveal leukocytosis, anemia, and raised lactate dehydrogenase level., ${ }^{47}$ Plain X-rays of the tumor site usually depict permeative destruction of the affected bone by the tumor mass along with onionskin periostitis, as seen in our case. MRI further helps in defining the tumor mass, involvement of the surrounding soft-tissue, and bone marrow metastasis. CT scan of the lungs and whole-body bone scan help in detection of metastasis at the lungs and bones, respectively. ${ }^{9}$ Common

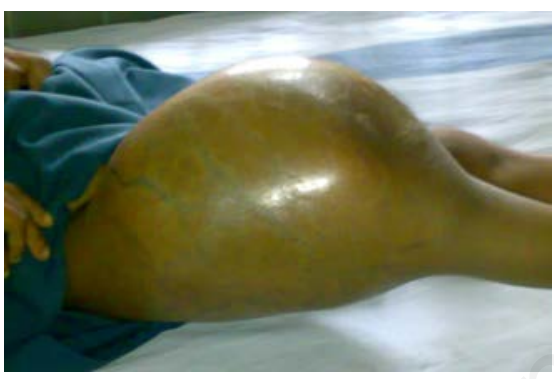

Figure 1. 5-year-old child with massive Ewing's sarcoma of the right lower limb.

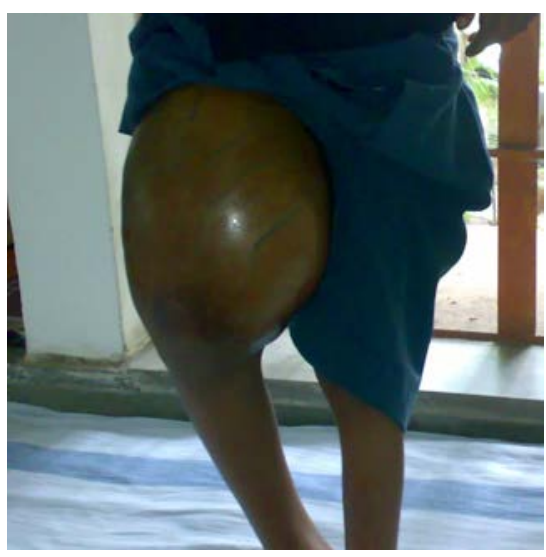

Figure 2. Large size of the tumor causing difficulty in standing. sites for metastasis of Ewing's sarcoma include lungs, bone, and bone marrow. ${ }^{4,9}$ Histologically, sheets of uniform small round tumor cells with round nuclei and little cytoplasm are seen, which may form a rosette. In over $90 \%$ cases of Ewing's sarcoma, CD99 is positive, as found in our patient. Other markers such as S-100, PGP9.5, and vimentin are also sometimes detected. ${ }^{4}$ CD99 expression is not specific for Ewing's sarcoma as it is also detected in several other malignancies such as acute lymphoblastic leukemia, lymphoma, and synovial sarcoma. Hence, identification of chromosomal translocations and chimeric genes specific to Ewing's sarcoma, as discussed earlier, via molecular techniques fluorescence in situ hybridization, and polymerase chain reaction is considered the gold standard of diagnosis. ${ }^{4,8}$ However, these molecular diagnostics are rarely utilized in economically-deprived countries due to their high cost.

Factors decreasing prognosis include presence of multiple metastasis, systemic symptoms (fever, weight loss), leukocytosis, increased lactate dehydrogenase levels, tumor size $(>8 \mathrm{~cm})$, tumor volume $(>200$ $\mathrm{mL}$ ), and site of tumor (pelvis). ${ }^{10}$ As our patient was positive for all the above mentioned prognostic factors, he was considered as a high-risk patient and neoadjuvant chemotherapy with alternating cycles of vincristine, adriamycin, actinomycin-D, cyclophosphamide (VDAC) and ifosfamide, etoposide (IE) was chosen., ${ }^{4,5,10}$ Recently, histopathological response to neoadjuvant chemotherapy (poor response defined as $>10 \%$ viable tumor cells as per Salzer-Kuntschik grading system) has emerged as the strongest prognostic factor overriding tumor size, tumor volume, or tumor location in localized Ewing's sarcoma. ${ }^{10}$ Stratification of the histopathological response to neoadjuvant chemotherapy also helps in grading and individualizing the chemotherapy, in the postoperative period.

The VDAC + IE regimen has a 5-year survival of $15-20 \%$ in high-risk patients with distant metastasis. ${ }^{5}$ Neoadjuvant chemotherapy eradicates any micrometastasis, reduces the size of the primary tumor to facilitate excision, and helps in selecting appropriate chemotherapy following surgery/radiotherapy. ${ }^{4} \quad$ Neoadjuvant chemotherapy for 9 weeks is usually followed by local treatment which includes surgery (amputation, limb salvage, or organ-sparing surgery) with or without radiotherapy. Ewing's sarcoma in children is treated with radiation doses ranging from 36-60 Gy. The choice of the local treatment is influenced by multiple factors such as age of the patient, site and size of the tumor, metastasis pattern, response to chemotherapy, preference of surgeon/patient, etc. Local therapy is usually followed by maintenance/consolidation therapy, which usually includes adjuvant chemotherapy for 44-48 weeks with or without radiotherapy to improve recurrence/relapse/survival rates. ${ }^{4,5,10}$

Despite intensive multimodal therapy, almost $70 \%$ of patients with advanced Ewing's sarcoma succumb to their illness. ${ }^{5,10}$ Novel molecular targets for Ewing's sarcoma being evaluated include drugs/biologicals inhibiting various types of tyrosine kinases such as insulin-like growth factor 1 receptor (R1507, cixutumumab, figitumumab, ganitumab, linsitinib), platelet-derived growth factor receptor (imatinib), epidermal growth factor receptor (gefitinib, erlotinib), and vascular growth factor receptor (cediranib, vandetanib, bevacizumab, sorafinib, pazopanib, axitinib, cabozantinib, regorafenib). ${ }^{1,2,8}$ ESW-FLI1-related targets include RNA helicase A inhibitors (YK-4-279, TK216), poly ADP ribose polymerase 1 inhibitors (olaparib, talazoparib, niraparib), histone deacetylase inhibitors (romidepsin, entinostat), lysine-specific demethylase inhibitors (HCI-2509), aurora kinase A inhibitors (alisertib), forkhead box $\mathrm{O}$ activators (methylseleninic acid), cholecystokinin inhibitors (devazepide), Gli protein inhibitors (arsenic trioxide), mammalian target of rapamycin inhibitors (deforolimus, irinotecan, temsirolimus, temozolomide), RNA polymerase II inhibitors (lubinectedin), cyclin dependent kinase inhibitors (abemaciclib), and protein kinase $\mathrm{C}$ beta inhibitors. ${ }^{2,6,8}$ Immunotherapy targets include development of EWS-FLI1 cancer vaccine, T cell and natural killer cell-based immunotherapies, cluster of differentiation 99 antibodies, IgG4 programmed cell death protein 1 antibodies (nivolumab), cytotoxic T-lymphocyte associated protein 4 antibodies (iplimumab), diganglioside GD2 antibodies (hu14, 18K322A), and tumor necrosis factor-related apoptosis-inducing ligand antibodies. ${ }^{2,8,10}$ Molecules acting on the bone tumor microenvironment such as osteoclast inhibitors (bisphosphonates) and receptor activator of nuclear factor kappa-B blockers (denosumab, bisphosphonates) are also being explored. ${ }^{3,10}$

Molecular diagnostics to detect abnormal expression of various genes/proteins and transcription factors/modulators, promoting tumorigenesis in Ewing's sarcoma patients, could help create a personalized chemotherapy regimen, consisting of novel targeted agents along with standard anticancer agents. ${ }^{8}$ However, as most of the above mentioned targeted therapies are in 
various phases of clinical trials, it would take several years more to determine their overall impact in improving the survival outcome, especially in advanced cases. ${ }^{2,8}$

\section{Conclusions}

Ewing's sarcoma with multiple distant metastasis has a dismal prognosis. Hence, early diagnosis and initiation of therapy helps improve survival/relapse/recurrence rates. Integrating conventional therapy with novel therapeutic agents holds a promise for high-risk patients with Ewing's sarcoma.

\section{References}

1. Jiang Y, Ludwig J, Janku F. Targeted therapies for advanced Ewing sarcoma family tumors. Cancer Treat Rev 2015;41:391-400.

2. Yu H, Ge Y, Guo L, Huang L. Potential approaches to the treatment of Ewing's sarcoma. Oncotarget 2017;8:5523-39.

3. Redini F, Heymann D. Bone tumor environment as a potential therapeutic target in Ewing sarcoma. Front Oncol 2015;5:279.

4. Ozaki T. Diagnosis and treatment of Ewing sarcoma of the bone: a review article. J Orthop Sci 2015;20:250-63.

5. Khanna N, Pandey A, Bajpai J. Metastatic Ewing's sarcoma: revisiting the 'evidence on the fence'. Indian J
Med Paediatr Oncol 2017;38:173-81.

6. Subbiah V, Kurzrock R. Ewing's sarcoma: overcoming the therapeutic plateau. Discov Med 2012;13:405-15.

7. Ross KA, Smyth NA, Murawski CD, Kennedy JG. The biology of Ewing sarcoma. ISRN Oncol 2013;2013:759725.

8. Pishas KI, Lessnick SL. Recent advances in targeted therapy for Ewing sarcoma. F1000Res 2016;5:2077-88.

9. Murphey MD, Senchak LT, Mambalam PK, et al. From the radiologic pathology archives: Ewing sarcoma family of tumors: radiological-pathological correlation. Radiographics 2013;33:803-31.

10. Biswas B, Bakhshi S. Management of Ewing sarcoma family of tumors: current scenario and unmet needs. World J Orthop 2016;18:527-38. 